\title{
Phase Regeneration of QPSK Signal in SOA using Single-stage, Wavelength Converting PSA
}

\author{
K. R. H. Bottrill ${ }^{1}$, R. Kakarla ${ }^{2}$, F. Parmigiani ${ }^{1}$, D. Venkitesh ${ }^{2}$, P. Petropoulos ${ }^{1}$
}

\begin{abstract}
We demonstrate, for the first time, all-optical phase regeneration of a quaternary phase shift keying (QPSK) signal through phase sensitive amplification (PSA) in nonlinear semiconductor optical amplifiers (SOAs), using a scheme only previously demonstrated in highly nonlinear fibre (HNLF). We make use of a highly tunable phase quantising scheme to circumvent some of the limitations imposed by the use of SOAs and show that it may function in either a conjugating or non-conjugating manner.
\end{abstract}

\section{INTRODUCTION}

One route towards all-optical regeneration of multiple data channels, which has enjoyed considerable attention in literature, has involved processing multiple channels in one medium. However, this approach is often marred by the difficulty in isolating the regenerated channels from each other during processing [1], [2], which either requires complicated optical schemes, or presents difficult engineering challenges, such as dispersion management in highly nonlinear fibre (HNLF). Processing multiple signals individually, each in their own spatially separated nonlinear medium, is perhaps a straight-forward alternative, even though it might not carry all of the scaling benefits that are often associated with all-optical signal processing. It may, however, still offer advantages over electronic signal processing in terms of latency, baud rate insensitivity, format flexibility, and broad bandwidth operation. It is likely that a process of miniaturisation and integration of the individual regenerators must be followed if such devices are to be made acceptably practical and compete with their electronic counterparts. Semiconductor optical amplifiers (SOAs) are particularly attractive for integration in an all-optical signal processing device, combining a nonlinear medium and an amplifier in a compact size on the order of millimetres. The reduced propagation length also provides further benefits over HNLF in terms of reduced latency.

Digital signal processing has progressed to the extent that the deployment of coherent receivers has become justifiable and, combined with bandwidth limitations imposed by components such as EDFAs and modulators, has spurred on investi-

\footnotetext{
${ }^{1}$ Optoelectronics Research Centre, University of Southampton, SO17 1BJ, UK.

${ }^{2}$ Indian Institute of Technology Madras, Chennai, India, 600036.

This research is sponsored by EPSRC grant EP/I01196X, The Photonics Hyperhighway.

Dr F. Parmigiani is supported by a Royal Academy of Engineering/EPSRC research fellowship.

The research leading to these results is supported by the People Programme (Marie Curie Actions) of the European Unions Seventh Framework Programme FP7/2007-2013/ under REA grant agreement n0318941.

The data for this work is accessible through the University of Southampton Institutional Research Repository (DOI:10.5258/SOTON/382591)
}

gation into complex, multi-levelled modulation formats to provide a cost effective way of increasing data throughput without upgrading transmission line components such as amplifiers and fibre. This movement towards complex transmission formats makes it desirable to demonstrate that all-optical signal processing can be achieved, not only upon simple binary formats, but more complicated ones as well [3]. However, although alloptical regeneration of on-off-keying [4] and binary phase shift keying [5], [6] has been demonstrated in SOAs, to date there has been no demonstration of all-optical regeneration of higher order signal formats, despite their demonstration in HNLF.

We have recently demonstrated that by combining the 3rd harmonic of a signal and its conjugate at a frequency independent from the original signal, we can achieve phase squeezing of a quaternary phase shift keyed (QPSK) signal for a large range of input pump and signal powers [7]. The ease of tunability of this scheme is particularly beneficial when the nonlinear medium to be used is an SOA, as the launch conditions it requires may be easily met. The use of this scheme in an SOA, allows us to demonstrate, for the first time in this medium, phase sensitive amplifier (PSA) based phase squeezing of a QPSK signal.

\section{CONCEPT}

Four-level phase quantisation with flat-topped quantisation steps may be achieved by coherently adding to a signal its conjugated 3rd phase harmonic with a magnitude ratio of $3: 1$, respectively [8], [9], and this is essentially the operating mechanism of the wavelength-converting idler-free scheme used in this demonstration. As shown in Figure 1-a, the wavelength converting idler-free scheme can be viewed as comprising the two underlying four wave mixing (FWM) operations shown in Figures 1-b and 1-c. The first uses a cascaded pump degenerate process commencing with an interaction between P1, a CW pump lying at $\omega_{P 1}$, and $\mathrm{S}$, a signal lying at $\omega_{S}$, and is used to generate the 3rd phase harmonic of the signal at $3 \omega_{S}-2 \omega_{P 1}$ (shown in Figure 1-b). Meanwhile, a non-degenerate FWM process occurs between P1, P2 (at frequency $\omega_{P 2}$ ) and $\mathrm{S}$, resulting in an idler lying at frequency $\omega_{P 2}+\omega_{P 1}-\omega_{S}$ which is the phase conjugate of the signal. Phase squeezing occurs when the idlers corresponding to phase conjugate and the third harmonic phase are generated at the same spectral location to facilitate coherent addition. This condition can be satisfied if $\mathrm{P} 2$ is chosen to lie at a frequency of $4 \omega_{S}-3 \omega_{P 1}$ and the pumps are suitably phase locked to the signal carrier. As the power of the signal conjugate generated at $3 \omega_{S}-2 \omega_{P 1}$ depends upon the power of P2, whilst the power 

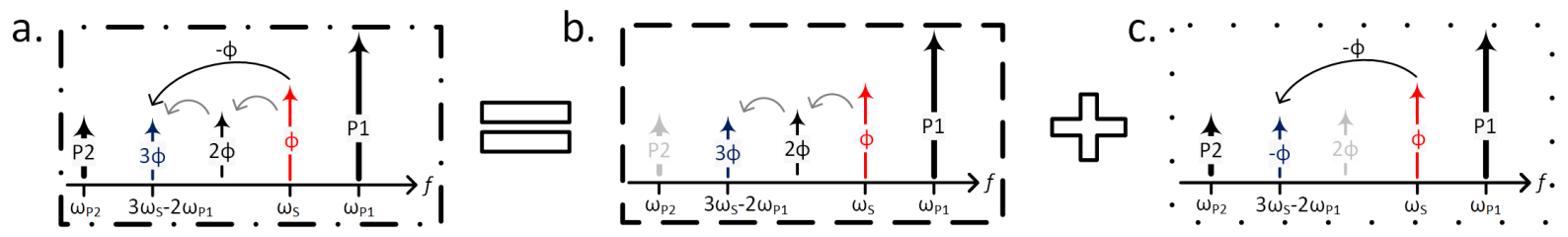

Fig. 1. Illustration of wavelength-converting idler-free QPSK regenerator and its two underlying simultaneous FWM stages. Curved arrows represent individual FWM interactions.

of the 3rd harmonic generated at the same location does not, the level of squeezing may be controlled simply by tuning the power of P2. As the two processes occur simultaneously, we can maximise the usage of the nonlinearity offered by the medium by making it perform two FWM tasks at once.

The wavelength-converting idler-free scheme has so far only been demonstrated in a manner which produces a phase conjugated output. However, it is also possible to obtain an unconjugated, albeit still wavelength converted output from the same system, simply by choosing the regenerated output that appears on the opposite side of the pump to the original input signal with frequency $4 \omega_{P 1}-3 \omega_{S}$. We take the opportunity to demonstrate this possibility by detecting both conjugated and unconjugated output, identified by labels $\bar{S}^{\prime}$ and $S^{\prime}$ in Figure 2. Due to the relative location of the pump and signal, conjugated output is red-shifted relative to the signal, whilst unconjugated output is blue-shifted. The conjugated output stems from the interference between conjugated signal photons (of phase $e^{-i \phi}$ ) and the 3rd harmonic $\left(e^{i 3 \phi}\right)$, whereas the unconjugated output is obtained through the interaction between unconjugated signal photons $\left(e^{i \phi}\right)$ and conjugated 3rd harmonic photons $\left(e^{-i 3 \phi}\right)$.

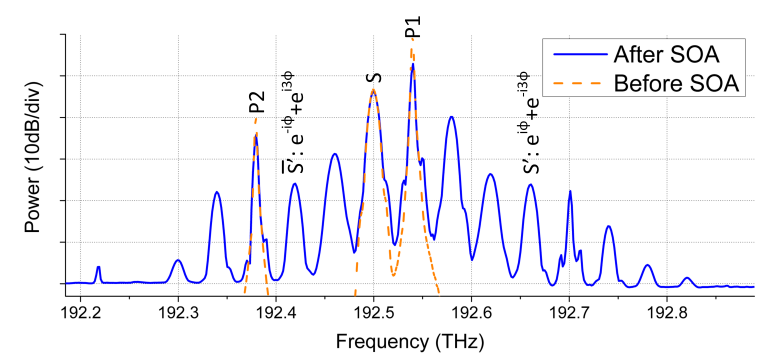

Fig. 2. Example of spectra before (dashed orange line) and after (solid blue line) FWM in SOA.

\section{EXPERIMENTAL SETUP}

Figure 3 provides a schematic of the experimental setup used in this study. Prior to the actual stage of phase regeneration, the setup can be seen to consist of two separate sections, a signal generation stage and a pump generation stage. Both stages are fed by the same $192.5 \mathrm{THz} \mathrm{CW}$ laser to lessen the challenge of phase tracking in this demonstration.

The signal generation stage consists of an IQ modulator which is used to impart a 10 GBaud non-return-to-zero QPSK signal onto the carrier and a phase modulator which is used to contaminate the signal with a controllable amount of broadband phase noise. The phase noise is generated by detecting amplified spontaneous emission (ASE) with a photo detector whose output is further amplified and fed to the phase modulator. Using the phase modulator we also add a sinusoidal phase dither to the signal used by the feedback system to lock the phase of the pumps to the signal [10].

Pumps are generated using an overdriven Mach-Zehnder modulator (MZM) to produce an optical frequency comb with a tone spacing of $40 \mathrm{GHz}$. This comb is passed through a programmable filter (PF) to select pumps $\mathrm{P} 1$ and $\mathrm{P} 2$ which lie at $192.54 \mathrm{THz}$ and $192.38 \mathrm{THz}$, respectively (see Figures 1 and 2), and enables control of their power. The pumps then pass through a fibre stretcher which is driven in a feedback loop (whose error signal is obtained from the dither applied previously) to ensure phase tracking of the signal carrier.

The pumps and signal are then combined using an optical coupler and launched into two cascaded, commercially available nonlinear SOAs (manufactured by Kamelian), each $0.5 \mathrm{~mm}$ long, with a saturation power of $5 \mathrm{dBm}$ and a gain recovery time of $25 \mathrm{ps}$, to undergo FWM. Two SOAs are used in cascade to improve the efficiencies of the FWM processes. After the SOAs, the phase squeezed outputs at $192.42 \mathrm{THz}$ (conjugated) and $192.66 \mathrm{THz}$ (unconjugated), are extracted using a tunable optical bandpass filter. Part of the output is passed onto the receiver, whilst a small percentage $(\approx 1 \%)$ is detected with a photodetector whose output contains the error signal used to drive the aforementioned fibre stretcher.

The receiver consists of a variable attenuator, an EDFA, an optical bandpass filter and an optical modulation analyser (OMA). As stated previously, if any two of P1, P2 and $\mathrm{S}$ are fixed in power, we may achieve any level of phase squeezing, simply by tuning the power of the remaing third wave. However, it is important to note that whilst the phase squeezing level achievable is independent of the combination of powers chosen, the optical signal to noise ratio (OSNR) is not. It is important to select a combination of powers which results in good OSNR whilst avoiding excessive self-phase modulation (SPM). We chose to study the level of phase noise reduction that could be achieved for a given $\mathrm{P} 1 \mathrm{~S}$ power ratio after phase squeezing had been maximised by tuning P2. Once we had found the P1:S power ratio that resulted in the greatest squeezing, we used it as the basis for further optimisation.

Due to the phase dependent gain of the scheme, a decrease in the phase noise of a signal is accompanied by an increase in its amplitude noise. For a given phase noise input to the regenerator, there will be an optimal level of phase squeezing to maximise the receiver sensitivity for a particular BER; if the phase squeezing is insufficient, then the benefits of the phase regenerator will not be fully realised, whilst if 


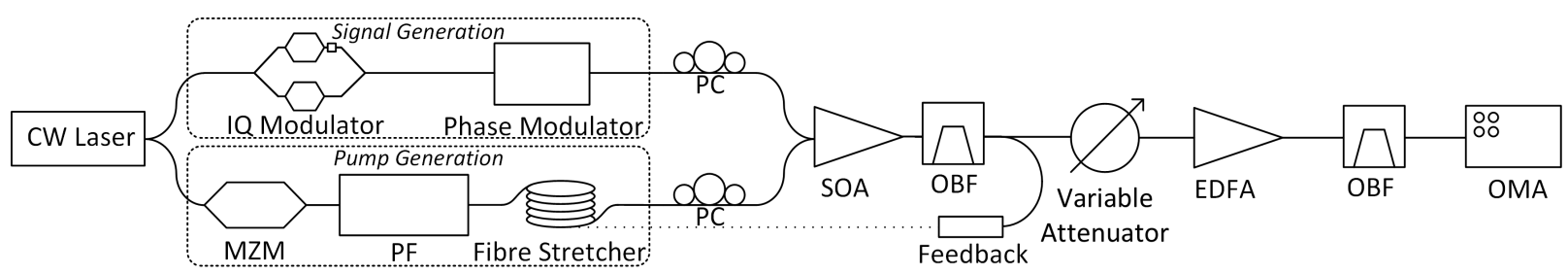

Fig. 3. Schematic of the wavelength-converting idler-free experimental set-up.

the phase squeezing is excessive, the increase in amplitude noise will outweigh the decrease in phase noise. We optimised the system to for the highest level of input phase noise for which we performed measurements, $12.2 \mathrm{deg}$. rms. The phase regenerator, with $\mathrm{P} 1: \mathrm{S}$ ratio determined above, was applied to the phase noise loaded signal, and the power of the pump, $\mathrm{P} 2$, tuned so as to minimise the OSNR required to obtain a BER of $10^{-3}$ (chosen as this is a well known forward error correction limit) for the unconjugated output. It should be noted that measurements of conjugated and unconjugated output result from the same optimisation - i.e. optimisation was not performed separately upon the conjugated output. All measurements presented in the results section are derived from the same optimisation; optimisation was performed only once. Using a drive current for the SOAs of $350 \mathrm{~mA}$ each, the following launch powers were determined through the optimisation process: $\mathrm{P} 1=9.7 \mathrm{dBm}, \mathrm{S}=-2.2 \mathrm{dBm}$ and $\mathrm{P} 2=-9.3 \mathrm{dBm}$.

\section{RESUlTS}

Firstly, we consider the effect the regenerator has upon the noise statistics of the signal. The black open diamonds in Figure 4-a show that the phase noise loading system does not contaminate the signal with magnitude noise, as desired. For the regenerated case (red and blue solid circles), we see that the output phase noise is very similar between the conjugated or unconjugated output and both exhibit a marginal upwards slope, indicating a phase transfer function with steps that are quite 'flat'. By comparing the regenerated phase noise curves to the unregenerated (linear) phase noise curve plotted with black circles, plotted for reference, we see that the system results in a reduction in phase noise for all input phase noises exceeding $5.5 \mathrm{deg}$. rms and for the highest input noise case of 12.2 deg. rms, the system results in a halving of phase noise. The output magnitude noise in all cases can be seen to be worse than the input magnitude noise, worsening as the input phase noise increases. This phase noise dependent increase in magnitude noise is expected from this type of phase squeezer [10] and is the result of phase dependent gain. The large increase in phase noise for low input phase noise levels is due to the ASE induced magnitude noise being converted into phase noise through nonlinear effects (such as SPM and crossphase modulation (XPM)) as well as the ASE itself, which we believe can be improved through the use of a longer SOA.

Constellation plots are presented in Figure 4-b before regeneration (middle row, yellow), and for both conjugated and unconjugated output, each for three different input phase noise cases. We see that the conjugated and unconjugated results

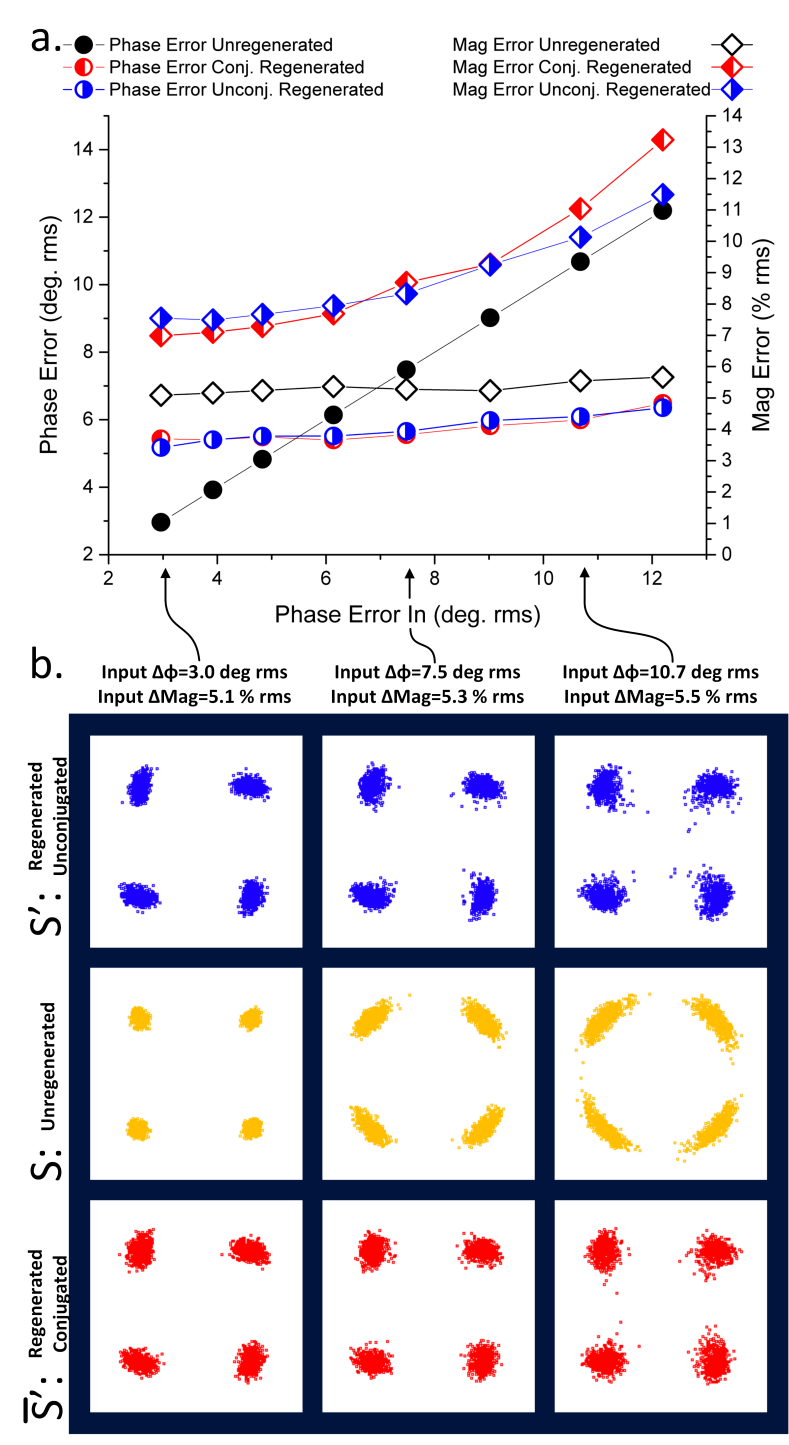

Fig. 4. a. Phase and amplitude noise statistics before regeneration and after regeneration for both the conjugated and unconjugated, regenerated output. b. Constellation plots for the three input phase noise levels studied for the unregenerated case, as well as both the conjugated and unconjugated, regenerated output.

match each other quite well, both showing deterioration of the signal in the case of low phase noise, but showing a clear reduction in phase noise for the higher input noise levels. We identify the following factors that are responsible for the increase in phase noise observed for low input phase noises. The poor OSNR of the pumps and consequent amplitude noise 
induces a nonlinear phase noise in the signal [11]. In order to avoid phase changes in the pump due to cross-gain modulation as well as the effects of SPM upon the signal, the P1:S ratio should ideally be as large as possible. However, the P1:S ratio used in our experiment could not be increased beyond $12 \mathrm{~dB}$ as this led to insufficient idler power and unacceptably low OSNR of the output. Thus the relatively small P1:S ratio in our experiments resulted in additional phase noise in the pump, further degrading the regenerated output constellations. If P1:S is to be increased without detriment to the OSNR, we must compensate for the loss of idler power through some other means, for instance by using a longer length of SOA.
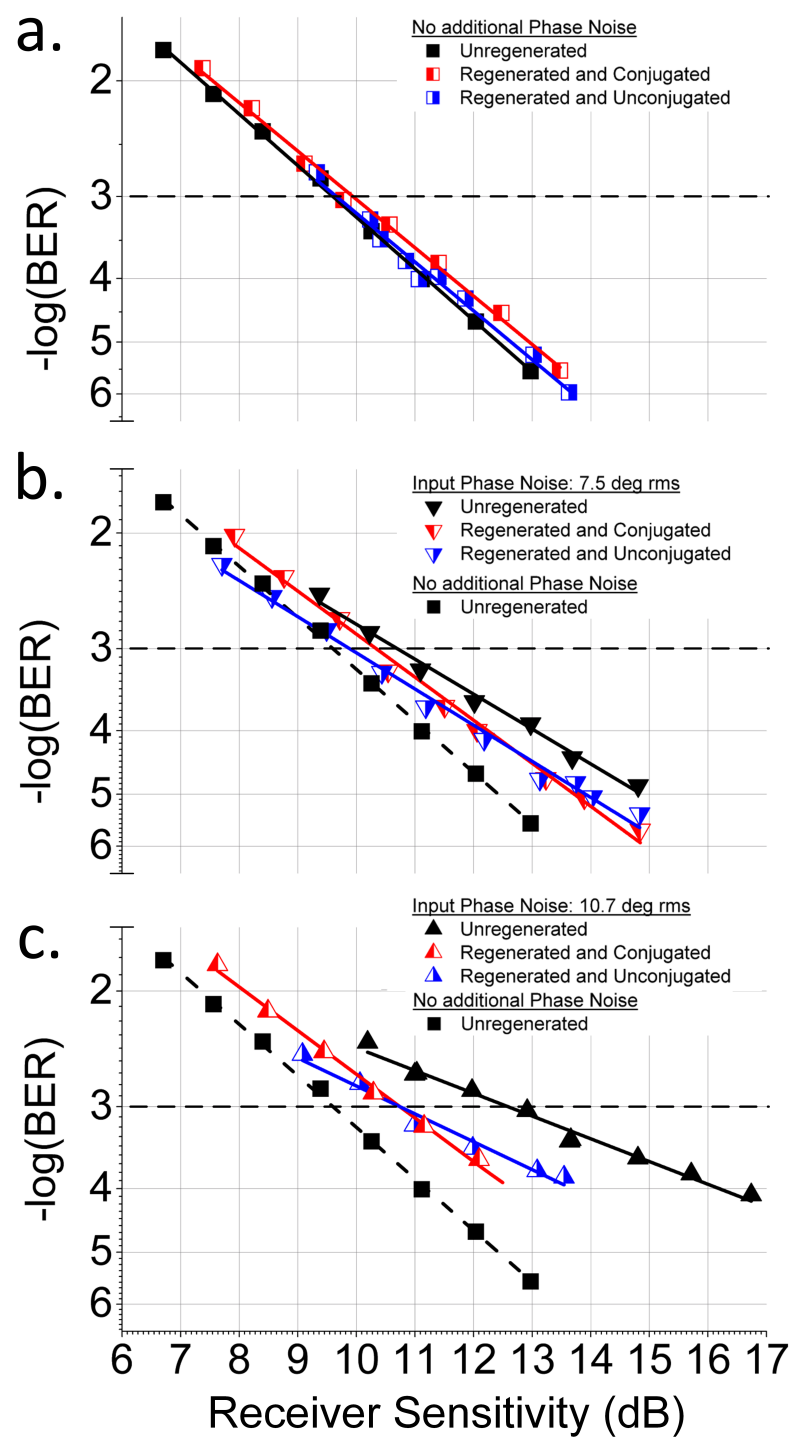

Fig. 5. Bit error ratio measurements for 3 input phase noise level cases.

Figure 5 provides BER curves corresponding to the three noise levels for which constellation plots were presented. These are obtained by loading the signal after regeneration with ASE to control the OSNR of the signal at the receiver. The results are separated into three graphs for clarity in Figure 5. For the lowest noise case (no additional noise), plotted with squares in Figures 5 -a, -b and -c, we see that regeneration is associated with a power penalty of less than $0.5 \mathrm{~dB}$, for both conjugated and unconjugated regeneration.

For an input phase noise of $\Delta \phi=7.5 \mathrm{deg}$. rms, plotted in Figure 5-b, receiver sensitivity is improved by $0.4 \mathrm{~dB}$ for a BER of $10^{-3}$ for the conjugated and $0.8 \mathrm{~dB}$ unconjugated output. For the highest phase noise considered, corresponding to an input phase noise of $\Delta \phi=10.7 \mathrm{deg}$. rms, plotted in Figure 5-c, receiver sensitivity is improved by $1.8 \mathrm{~dB}$ at $10^{-3}$ for both the conjugated and unconjugated case.

Gradient changes in BER curves after phase squeezing are due to the distribution of noise in phase and amplitude. PSK formats of order 4 or more are more sensitive to phase noise than amplitude noise, which becomes more important for low OSNRs. The differing gradients between the conjugated and unconjugated output is because the two outputs have different ratios of signal to 3rd harmonic photons, being the result of different FWM processes, resulting in different levels of squeezing and hence different noise distributions.

\section{CONClusion}

We have demonstrated phase regeneration of a QPSK signal in SOA, obtaining a $1.8 \mathrm{~dB}$ improvement in receiver sensitivity for a BER of $10^{-3}$ for a signal contaminated with a high level of phase noise. We demonstrated both conjugating and nonconjugating operation of the wavelength-converting idler-free scheme as a proof of principle. We believe improved results could be obtained by improving the OSNR of the pumps, operating the SOAs in deep saturation to reduce ASE and using a longer length of SOA medium in order to increase the power of the FWM outputs.

\section{REFERENCES}

[1] F. Parmigiani et al., "Progress in Multichannel All-Optical Regeneration Based on Fiber Technology," Selected Topics in Quantum Electronics, IEEE Journal of, vol. 18, no. 2, pp. 689-700, March 2012.

[2] M. Vasilyev, T. I. Lakoba, and P. G. Patki, "Multiwavelength All-Optical Regeneration," in $O F C / N F O E C, 2008$, p. OWK3.

[3] S. Namiki, T. Hasama, and H. Ishikawa, "Optical signal processing for energy-efficient dynamic optical path networks," in ECOC, Sept 2010, pp. $1-6$.

[4] M. Nielsen, B. Olsson, and D. Blumenthal, "Pulse extinction ratio improvement using SPM in an SOA for OTDM systems applications," Photonics Technology Letters, IEEE, vol. 14, no. 2, pp. 245-247, Feb 2002.

[5] R. Manning et al., "SOAs for Phase-Based Optical Signal Processing," in Photonics Society Summer Topical Meeting Series, 2014 IEEE, July 2014, pp. 110-111.

[6] A. D. Ellis and S. Sygletos, "Phase Sensitive Signal Processing using Semiconductor Optical Amplifiers," in OFC/NFOEC, 2013, p. OW4C.1.

[7] K. R. Bottrill et al., "FWM-based, Idler-free Phase Quantiser with Flexible Operating Power," in $O F C, 2015$, p. W4C.3.

[8] J. Kakande et al., "Multilevel quantization of optical phase in a novel coherent parametric mixer architecture," Nature Photonics, vol. 5, no. 12 , pp. $748-752$, Dec. 2011.

[9] T. Kurosu et al., "Phase regeneration of phase encoded signals by hybrid optical phase squeezer," Opt. Express, vol. 22, no. 10, pp. 12 177-12 188, May 2014.

[10] R. Slavík et al., "All-optical phase and amplitude regenerator for next-generation telecommunications systems," Nature Photonics, vol. 4, no. 10 , pp. 690-695, Oct. 2010.

[11] A. Anthur et al., "Impact of Nonlinear Phase Noise on All-Optical Wavelength Conversion of 10.7-GBaud QPSK Data Using Dual Correlated Pumps," Quantum Electronics, IEEE Journal of, vol. 51, no. 4, pp. 1-5, April 2015. 